\title{
A High Efficiency Cosmic Ray Veto for the Mu2e Experiment
}

\section{E. Craig Dukes ${ }^{1 *}$}

University of Virginia

Charlottesville, VA, USA

E-mail: craigdukes@virginia.edu

\section{Ralf Ehrlich*}

University of Virginia

Charlottesville, VA, USA

E-mail: ehrlich@virginia.edu

The Mu2e experiment will study charged lepton flavor violation by searching for a neutrinoless, coherent conversion of a $\mu^{-}$to an $\mathrm{e}^{-}$. Such a process will result in an electron of $105 \mathrm{MeV}$ energy. A major background source comes from cosmic-ray muons which can produce electrons that mimic these conversion electrons at a rate of approximately one per day. An active veto detector surrounding the apparatus will be used to detect incoming cosmic-ray muons.

38th International Conference on High Energy Physics 3-10 August 2016

Chicago, USA

\footnotetext{
${ }^{1}$ Speaker

*On behalf of the Mu2e collaboration

(C) Copyright owned by the author(s) under the terms of the Creative Commons

Attribution-NonCommercial-NoDerivatives 4.0 International License (CC BY-NC-ND 4.0).
} 


\section{The Mu2e Experiment}

Mu2e will search for charged lepton flavor violation through the conversion process $\mu \mathrm{N} \rightarrow \mathrm{e}^{-\mathrm{N}}$ in the field of an Al nucleus [1]. Observation of such a process would be unambiguous evidence of physics beyond the standard model. The signature is a single delayed $105 \mathrm{MeV}$ electron. Protons of $8 \mathrm{GeV}$ produce a bunched beam of 39 million muons delivered every $1695 \mathrm{~ns}$ on thin $\mathrm{Al}$ stopping target foils. In a three-year run a single-event sensitivity of $2.9 \cdot 10^{-17}$ is expected: 10,000 times better than the current limit.

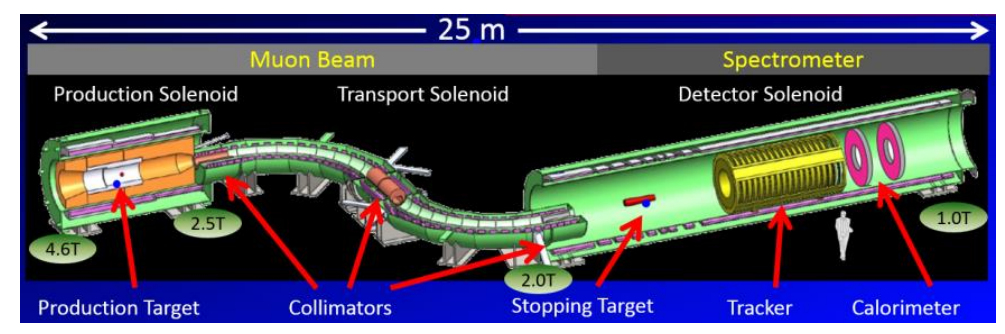

Figure 1: The layout of the Mu2e experiment. A proton beam hits the production target to produce muons which are transported through the transport solenoid to the stopping target. Electrons produced at the stopping target are measured by the tracker and calorimeter.

\section{Cosmic Ray Induced Backgrounds}

Cosmic-ray muons rain down upon the detector at a rate of about 15,000 per second. Roughly once per day one will produce a conversion-like event, either by the muon itself being misidentified as an electron, or it decaying into an electron, or it knocking off a $105 \mathrm{MeV}$ electron in material in the Detector Solenoid. A simulation of one such event is shown in Figure 2.

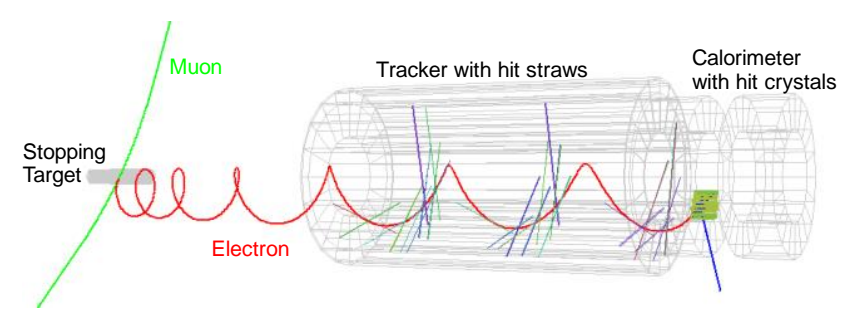

Figure 2: Simulation of a muon knocking out a delta-electron from a stopping target foil which spirals into the tracker and hits the calorimeter. The event appears identical to a real conversion electron.

To determine the extent of coverage of the Cosmic Ray Veto, and to estimate the required veto efficiency, we have simulated 3.7 trillion cosmic-ray muons, four times the total live time of the experiment, and in certain areas where there are unavoidable gaps in coverage, over 250 times the total live time. To keep the background to less than 0.1 events over the course of the run an overall veto efficiency of $99.99 \%$ is needed.

\section{The Cosmic Ray Veto}

The Cosmic Ray Veto surrounds the Detector Solenoid. It is composed of 4 layers of extruded polystyrene scintillator counters, with $\mathrm{TiO}_{2}$ coating, and embedded wavelength shifting fibers, read out by SiPM photodetectors. A track stub in at least 3 out of 4 layers, localized in time and space produces a veto in the offline analysis. The design is driven by the need for 
excellent muon veto efficiency (99.99\%), large area, small gaps, high-background rates, access to the electronics, and operation in a high magnetic field. Design of the Cosmic Ray Veto is

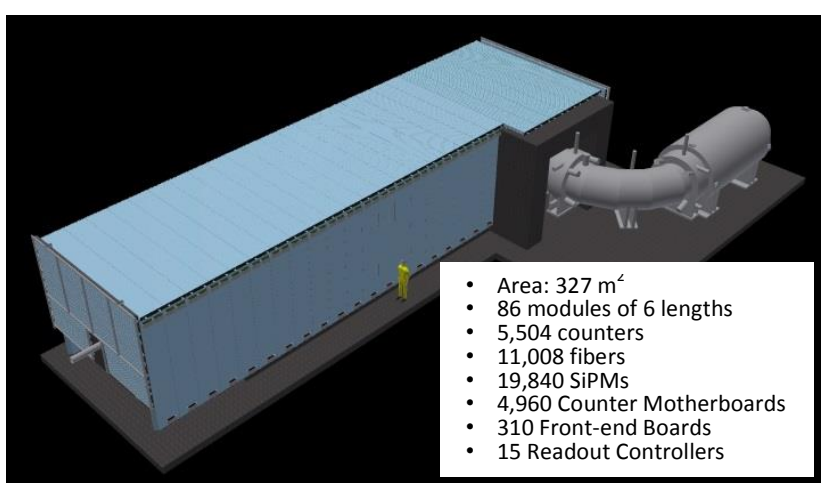
almost complete, prototypes of all components have been built, and fabrication will start in late 2017.

Figure 3: The cosmic ray veto surrounding the detector solenoid.

\section{The Counter}

The fundamental element of the Cosmic Ray Veto is the counter, of which there are 5504. Counters are $50 \times 20 \times 900 \ldots 6600 \mathrm{~mm}^{3}$ in size, are extruded at the FNAL-NICADD facility, and have two $1.4 \mathrm{~mm}$ diameter wavelength shifting fibers embedded in channels. Each fiber is read out on both ends by a $2 \times 2 \mathrm{~mm}^{2}$ SiPM. Flasher LEDs on each counter end are used for calibration and a thermometer measures the temperature of the SiPMs, which is not controlled; rather the SiPM bias is adjusted based on the temperature. Two extrusions are glued together to form a di-counter, served by one counter motherboard.

The Fiber Guide Bar funnels the four fibers into narrow channels at its SiPM end. Wells in the SiPM Mounting Block seat the SiPMs, which are mounted on tiny carrier boards. The SiPM carrier boards are connected to the Counter Motherboard via spring-loaded pogopins, which gently push the SIPMs against the fiber ends. An HDMI cable connects the Counter Motherboard to the Front-end Board, which amplifies and digitizes the SiPM signals, as well as providing the SiPM bias, and flashing the LED, and reading out the thermometer.
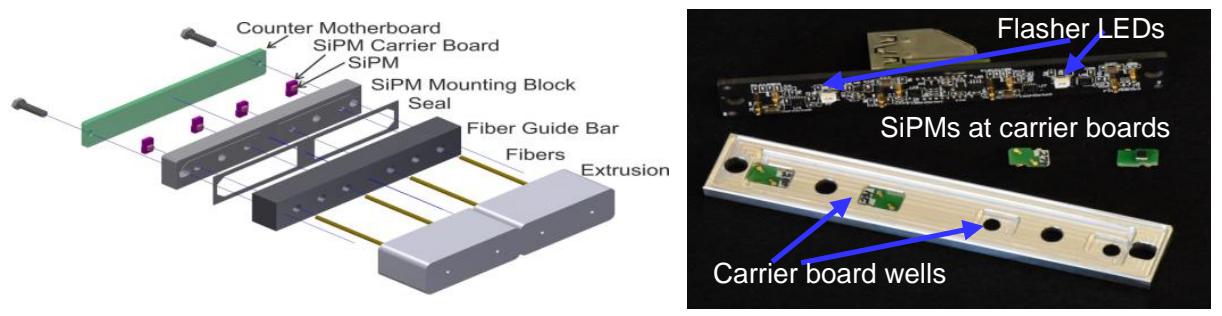

Figure 4: The readout end of a di-counter.

\section{The Module}

The fundamental mechanical element of the Cosmic Ray Veto is the module, of which 86 are needed, all identical except for their lengths. Each module consists of 4 layers of counters (with 16 counters per layer), and 3 layers of $\mathrm{Al}$ plates sandwiched between them. The layers are offset to minimize the effect of projective gaps.

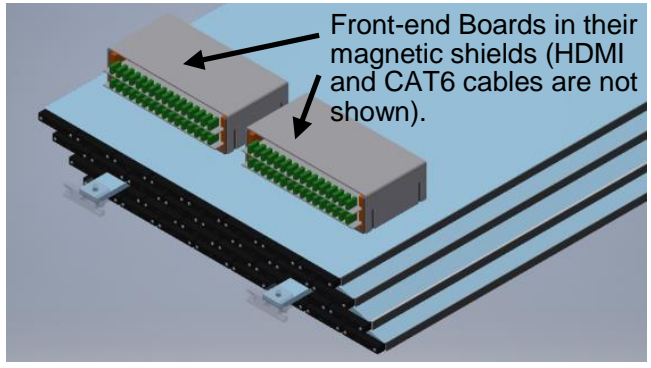

Figure 5: A CRV module. 


\section{Electronics}

There are four components: (1) surface mounted SiPMs, (2) Counter Motherboards (CMB), (3) Front-end Boards (FEB), and (4) Readout Controllers (ROC). All use COTS parts, the heart being the $80 \mathrm{MHz}$ ultrasound octal amplifier/ADC on the FEBs. Short ( 1 m) HDMI cables take 64 SiPM signals from the CMBs to the FEBs, where they are amplified, digitized, zero-suppressed, time stamped, and buffered (0.380s bunch period). The FEBs send data over CAT6 cables to ROCs in the control room, each ROC handles up to 24 FEBs. ROCs send their data to the Data Transfer Controllers and the DAQ. The ROCs power the FEBs through Ethernet.

\section{Performance}

In order to veto incoming muons with an inefficiency of $0.01 \%$, single layer inefficiencies must be no more than $0.5 \%$. Extensive simulations with a soup-to-nuts MC have shown that to meet this efficiency requirement the SiPM photoelectron yield must be $25 \mathrm{PE} / \mathrm{cm}$ (for a normally incident muon $1 \mathrm{~m}$ from the SiPM). Test-beam results give $42 \mathrm{PE} / \mathrm{cm}$, producing an adequate safety factor.

\section{Rates in the Cosmic Ray Veto}

The Cosmic Ray Veto sits on 1-m thick concrete shielding needed to reduce the neutron and gamma rates from the intense primary proton and muons beams. The average SiPM hit rate is $44 \mathrm{kHz}$ during the live gate. Neutron rates, shown in Figure 6, are within limits. Deadtime is estimated to be $\sim 5 \%$.

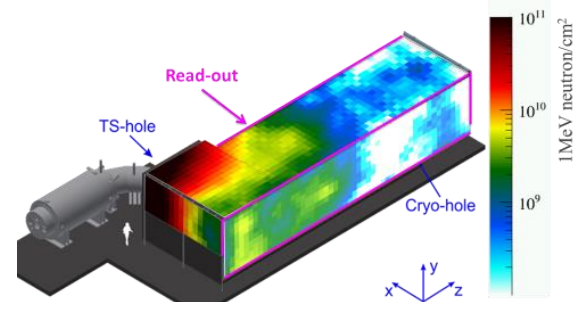

Figure 6: Neutron rates at the CRV.

\section{Acknowledgements}

We are grateful for the vital contributions of the Fermilab staff and the technical staff of the participating institutions. This work was supported by the US Department of Energy; the Italian Istituto Nazionale di Fisica Nucleare; the US National Science Foundation; the Ministry of Education and Science of the Russian Federation; the Thousand Talents Plan of the Republic of China; the Helmholtz Association of Germany; and the EU Horizon 2020 Research and Innovation Program under the Marie Sklodowska-Curie Grant Agreement No.690385. Fermilab is operated by Fermi Research Alliance, LLC under Contract No. De- AC02-07CH11359 with the US Department of Energy.

\section{References}

[1] L. Bartoszek, et al., Mu2e Technical Design Report, arXiv:1501.05241 [physics.ins-det]. 